\title{
Concerning certain minimal cover refineable spaces ${ }^{(1)}$
}

\section{by}

\section{U. J. Christian (Gaithersburg)}

The concept of minimal cover refineable spaces was first used by R. Arens and J. Dugundji. This paper extends their results to show both additional properties which imply minimal cover refineability and additional properties which are implied by minimal cover refineability.

In the course of the research for this paper, some properties of dense subspaces of certain minimal cover refineable spaces were noted. In particular, it was noted that every Nagata space contains a dense metrizable subspace.

1. Introduction. The property of minimal cover refineability will be seen to be a very weak property - one of the basic topological building blocks. It is hoped that this paper will encourage other mathematicians to attempt to link this basic concept with additional basic concepts in new and significant ways.

This paper is divided into three sections. This first section will define minimal cover refineability and identify some spaces which possess this property. A space which does not possess this property is also described.

Section 2 is primarily concerned with dense subspaces of certain minimal coves neable spaces, and, in particular, with dense metrizable subspaces. Of ticular interest is the result that each Nagata space contains a de metrizable subspace. This section also discusses a relation betwee: in weight of a space and Souslin's number.

Section 3 gives properties that force subsets of minimal cover refineable spaces to be minimal cover refineable.

In this paper, "space" means "a $T_{3}$ topological space", the word "cover" means "open cover," and a refinement is assumed to be composed of the same kind of elements (i.e., open or closed) and cover the same set as the collection which is refined.

(1) This paper is based upon the author's doctoral dissertation at the University of Houston, written under the direction of D. R. Traylor.

15 - Fundamenta Mathematicae T. LXXVI 
The statement that $K$ is a minimal cover of the point set $M$ means that $K$ is an open cover of $M$ and if $g$ is an element of $K$, then $\{h: h \in K$ and $h \neq g\}$ is not a cover of $M$.

The statement that a point set $M$ is minimal cover refineable means that if $K$ is an open cover of $M$, then there is a refinement of $K$ which is a minimal cover of $M$.

Examples of minimal cover refineable spaces are metacompact spaces (as proved by Arens and Dugundji [4]) and $F_{\sigma}$-screenable spaces (cf $[3 \tilde{5}]$ ).

THEOREM 1.1. In an $\mathrm{s}_{1}$-compact space, the following are equivalent: (a) Lindelöf, (b) metalindelöf, (c) paralindelöf, (d) hypolindelöf, (e) screenable, (f) metacompact, (g) $F_{\sigma}$-screenable, (h) paracompact, (i) hypocompact, (j) $\sigma$-(any prior), (k) minimal cover refineable.

G. Aquaro has shown that (b) implies (a) [3]. (His argument seems unduly tedious. It need only be noted that if $C$ is an open cover of a space $S$, then the collection $\left\{g_{p}: p \in S, g_{p}=\bigcup\{h: h \in C\right.$ and $\left.h \cap p \neq \emptyset\}\right\}$ has a minimal subcover.) By Morita [30], (a) implies (i). The balance of the argument should be obvious.

By Theorem 1.1, the countable ordinals with the order topology, commonly denoted by $[0, \Omega)$ is an example of a space which is not minimal cover refineable.

2. Separability and related concepts. This section will be concerned with dense subsets of semi-stratifiable spaces and, in particular, with dense, metrizable subspaces.

Several papers of the last decade have dealt with dense metrizable subspaces of Moore spaces. In [36], J. N. Younglove established that each complete Moore space contains a dense metrizable subspace. B. Fitzpatrick showed a Moore space that has no dense metrizable subspace in [14] and gave additional conditions for dense metrizable subspaces of Moore spaces. In [15], B. Fitzpatrick proved that a normal Moore space which is not a counterexample of type $D$ has a dense metrizable subspace. In [32], C. W. Proctor showed that if a normal locally connected Moore space has a base with the property that the space is collectionwise normal with respect to each discrete subset that is contained in the boundary of some base element, then the space contains a dense metrizable subspace.

The research into the conditions in which a semi-stratifiable space has a dense metrizable subspace was undertaken at the suggestion of D. R. Traylor.

Two papers that have theorems dealing with dense subspaces are in the bibliography [2], [10].

The statement that a space $S$ is semi-stratifiable means that there is a function $G$ from the product of the collection of closed sets of $S$ with the natural numbers into the collection of open sets in $S$ such that (i) $\bigcap_{i=1}^{\infty} G(A, n)=A$ for each closed set $A$ and (ii) $G\left(A_{1}, n\right) \subset G\left(A_{2}, n\right)$ whenever $A_{1} \subset A_{2}$.

A number of papers have dealt with stratifiable and semistratifiable spaces. Material on these spaces may be found in [7], [9], [11], [12], [17], [21], [31]. Arhangel'skí calls stratifiable' spaces laced and discusses them in [5].

The statement that a cardinal $m$ is $s_{0}-r e g u l a r$ means that the cardinal is not the sum of countably many cardinals, each less than $\mathrm{m}$.

The following definitions were taken from [23]:

If $\mathrm{m}$ is a cardinal, the point set $N$ is said to be strongly $\mathrm{m}$-separable provided that there exists an $H$ such that (1) $H \subset N \subset \bar{H}$ and (2) either $H$ is countable or its cardinality is less than $m$.

If $m$ is the cardinality of a point set $N$ and $N$ is strongly $m$-separable, then $N$ is said to be semi-separable.

The following theorem was proved after discovering that semistratifiable spaces are minimal cover refineable. The argument used illustrates how a minimal cover refinement of an open cover for a semistratifiable space was built.

THEOREM 2.1. Every semi-stratifiable space $S$ contains a dense $\sigma$-discrete point set. Further, if $\mathrm{m}$ is the cardinality of $\mathrm{S}$, then if (1) $\mathrm{m}$ is an $\mathrm{s}_{0}$-regular cardinal and $S$ is $\mathrm{m}$-compact, (2) $\mathrm{t}<\mathrm{m}$ and $\mathrm{S}$ is $\mathrm{n}$-compact, or $(3) \mathrm{m}<\mathrm{s}_{1}$, then $S$ is semi-separable and hereditarily strongly $\mathrm{m}$-separable.

Proof. Let $G$ be a function from the product of the collection of closed subsets of $S$ with the natural numbers into the collection of open sets in $S$ such that (i) $\bigcap_{n=1}^{\infty} G(A, n)=A$ for each closed set $A$; (ii) $G\left(A_{1}, n\right)$ $\subset G\left(A_{2}, n\right)$ whenever $A_{1} \subset A_{2}$. Well-order the points of $\boldsymbol{S}$. For each integer $m>0$, do the following: For each point $p$ of $S$, there is an integer $I>0$ such that $p \notin G(S \backslash G(p, m), I)$. Associate $I$ with $p$.

For each integer $i>0$, let $W_{i}=\{p: i$ is associated with $p\}$.

Since $S$ is well-ordered, there is a first element in the subcollection $W_{1}$, say $P_{11}$ (assuming, of course, that $W_{1}$ is not empty). Let $O_{11}$ $=G\left(P_{11}, m\right)$.

Let $P_{12}$ be the first point of the well-ordered collection $W_{1}$ which is not a point of $O_{11}$. Let $O_{12}=G\left(P_{12}, m\right) \cap G\left(S \backslash O_{11}, 1\right)$.

After each initial segment, let $P_{1 \alpha}$ be the first point of the wellordered collection $W_{1}$ which is not a point of any of the $O_{1 B}$ already constructed. Let $O_{1 \alpha}=G\left(P_{1 \alpha}, m\right) \cap G\left(S \backslash \bigcup_{\beta<\alpha} O_{1 \beta}, 1\right)$. 
For each integer $i>1$, let $P_{i 1}$ be the first point of the well-ordered collection $W_{i}$ which was not covered by any of the $O_{j \beta}, j<i$, already constructed. Let $O_{i 1}=G\left(P_{i 1}, m\right) \cap \bigcap_{i=1}^{i} G\left(S \underset{\substack{h<i \\ h_{i}}}{\bigcup} O_{h \beta}, j\right)$.

After each initial segment, let $P_{i a}$ be the first point of the well-ordered collection $W_{i}$ which is not a point of any of the $O_{j \beta}, j \leqslant i$, already constructed. Let

$$
o_{i a}=G\left(P_{i \alpha}, m\right) \cap \bigcap_{j=1}^{i} G\left(S \backslash\left(\bigcup_{\substack{h<i \\ \beta}} O_{h \beta} \cup \bigcup_{\beta<\alpha} O_{i \beta}\right), j\right) .
$$

Let $Q_{m}=\left\{P_{11}, P_{12}, P_{13}, \ldots, P_{1 \Omega}, \ldots, P,{ }_{i 1}, \ldots\right\}$. Note that $Q_{m}$ is discrete since $\left\{O_{11}, O_{12}, O_{13}, \ldots, O_{1 \Omega}, \ldots, O_{i 1}, \ldots\right\}$ covers $S$.

Let $p \in S$ and $R$ any open set containing $p$. There is an integer $I>0$ such that $p \notin G(S \backslash R, I)$. Therefore, $R \cap Q_{I} \neq \emptyset$. Hence, the collection $\left\{Q_{i}\right\}$ is the dense $\sigma$-discrete point set required.

The collection $\left\{Q_{i}\right\}$ demonstrates that if the hypothesis is met, $S$ is strongly $\mathrm{m}$-separable and, thus, semi-separable. It will now be shown that $S$ is hereditarily strongly $\mathrm{m}$-separable. Let $N$ be any subset of $S$. Use the construction above to generate $Q_{m}(N)=\left\{P_{11}(N), P_{12}(N), P_{13}(N), \ldots\right.$ $\left.\ldots, P_{1 \Omega}(N), \ldots, P_{i 1}(N), \ldots\right\}$ for each integer $m>0$ except that the points of $N$ are well-ordered instead of $S$ and, so, the elements of $\left\{W_{i}\right\}$ contain only points of $N$. Note that for each integer $m>0$, the collection $Q_{m}(N)$ can be decomposed into countably many discrete collections; i.e., $\left\{P_{11}(N), P_{12}(N), P_{13}(N), \ldots, P_{1 \Omega}(N), \ldots\right\}, \quad\left\{P_{21}(N), P_{22}(N), \ldots\right\}, \quad\left\{P_{31}(N)\right.$, $\left.P_{32}(N), \ldots\right\}, \ldots$ Thus $N$ is strongly $\mathrm{m}$-separable.

An example of a space which is compact but not semi-separable is the space $[0, \Omega)$. Separability does not imply the property of hereditary separability nor $s_{1}$-compactness even in a Moore space; McAuley provided an example of such a space [27].

A space is collectionwise Hausdorff if every discrete subset can be covered by a collection of disjoint open sets such that no two points of the discrete subset are contained in an element of the collection.

THeоRем 2.2. Every collectionwise Hausdorff normal semi-stratifiable space contains a dense paracompact subspace.

Proof. Let $S$ be a space satisfying the hypothesis and let $\left\{Q_{i}\right\}$ be a countable collection of discrete point sets such that $\bar{U}\left\{Q_{i}\right\}=S$. For each integer $i>0$, let $O_{i}$ be a collection of disjoint open sets which covers $Q_{i}$ and refines $C$, an arbitrary open cover of $\bigcup\left\{Q_{i}\right\}$. Let $H_{i}$ and $K_{i}$ be disjoint open sets containing $Q_{i}$ and $S \backslash O_{i}^{*}$, respectively. Let $O_{i}^{\prime}$ $=\left\{g: g^{\prime} \in O_{i}\right.$ and $\left.g=g^{\prime} \cap H_{i}\right\}$. The collection $\left\{O_{i}^{\prime}\right\}$ contains countably strongly screenable and, thus, paracom. This demonstrates that $U\left\{Q_{i}\right\}$ is
The statement that a space $S$ is basically metacompact means that if $B$ is a basis for $S$, then every open cover of $S$ has a refinement consisting of elements of $B$ which is point-finite. The statement that a space is basically minimal cover refineable means that if $B$ is a basis for the space, then every open cover of the space has a refinement consisting of elements of $B$ which is a minimal cover of the space.

The hypothesis of the following theorem is almost strong enough to give a dense metrizable subspace.

THEOREM 2.3. Every metacompact semi-metrizable space contains a dense basically metacompact (and, thus, basically minimal cover refineable) subspace with a point-regular basis.

Proof. Let $S$ be a space satisfying the hypothesis. Since a semimetrizable space is semi-stratifiable, there is a countable collection, $\left\{Q_{i}\right\}$, of discrete point sets such that $\overline{U\left\{Q_{i}\right\}}=S$. For each integer $i>0$, let $O_{i}$ be a point-finite open cover of $Q_{i}$ which refines the collection $\left\{g_{p}: p \in Q_{i}\right.$ and $\left.g_{p}=\mathbb{S} \backslash\left(Q_{i} \backslash p\right)\right\}$. Let $O_{1}^{\prime}=O_{1}$. For each integer $i>1$, let $O_{i}^{\prime}=\left\{g: g^{\prime} \in O_{i}\right.$ and $\left.g=g^{\prime} \backslash \bigcup_{j=1}^{i-1} Q_{j}\right\}$. Clearly, $\left\{g: i>0, g \in O_{i}^{\prime}\right\}$ is a pointfinite cover of $\bigcup\left\{Q_{i}\right\}$. Every subcollection of $\left\{g: i>0, g^{\prime} \in O_{i}^{\prime}, p \in Q_{i}\right.$, and $\left.p \in g \subset g^{\prime}\right\}$ covering $\bigcup\left\{Q_{i}\right\}$ contains a point-finite cover of $\bigcup\left\{Q_{i}\right\}$. Then $\bigcup\left\{Q_{i}\right\}$ is basically metacompact. By Arens and Dugundji [4], $\bigcup\left\{Q_{i}\right\}$ is basically minimal cover refineable.

It will now be shown that $\bigcup\left\{Q_{i}\right\}$ has a point-regular basis. Since $S$ is first countable, let $\left\{h_{p i}\right\}$ be a countable basis for each point $p$ of $S$. Let $O_{i j}^{\prime}=\left\{g: g^{\prime} \in O_{i}^{\prime}, p \in g^{\prime} \cap Q_{i}, g=g^{\prime} \cap \bigcap_{k=1}^{j} h_{p k}\right\}$. For each point $p$ of $\bigcup\left\{Q_{i}\right\}$, there is an integer $J>0$, such that if $j>J$, only one element of the collection $\left\{g: i>0, g \in O_{i j}^{\prime}\right\}$ contains $p$. Therefore, the collection $\left\{g: i, j>0, g \in O_{i j}^{\prime}\right\}$ is a point-regular basis for $\bigcup\left\{Q_{i}\right\}$.

The proof of the following theorem is a combination of the techniques developed to prove Theorems 2.2 and 2.3 and is not shown since the argument is so similar to that used for those two theorems.

THEOREM 2.4. Every collectionwise Hausdorff semi-metrizable space contains a dense basically metacompact (and, thus, basically minimal cover refineable) subspace with a point-regular basis.

The following theorem has been argued in the proof for Theorems 2.3 and 2.4.

THEOREM 2.5. If a semi-stratifiable space is either collectionwise Hausdorff or metacompact, it contains a dense basically metacompact (and, thus, basically minimal cover refineable) subspace.

THEOREM 2.6. Every collectionwise Hausdorff normal semi-metrizable. space contains a dense metrizable subspace. 
Proof. By the arguments used to establish Theorems 2.2 and 2.4, the space contains a dense paracompact subspace with a point-regular basis. By Alexandroff [1], this dense subspace is metrizable.

Since a Nagata space is a first countable stratifiable space [9], the following theorem is true:

THeOREM 2.7. Every Nagata space contains a dense metrizable subspace.

A definition of weight may be found in [13]; a definition for density (or character density) may be found in [8].

The Soustin number of a space is the upper bound of the cardinal numbers $m$ such that there exists in the space a collection of nt not empty disjoint open subsets.

The following theorem may prove useful in investigating the weight of a space. The theorem is an application of Theorem 2.1.

THEOREM 2.8. If $\mathrm{m}$ is an $\mathrm{s}_{0}$-regular cardinal and $\mathrm{m}$ is the density of a semi-stratifiable space $S$, then there is a discrete point set in $S$ with cardinality $\mathrm{m}$.

The reader should convince himself that the following theorem is true:

THEOREM 2.9. Every hereditarily separable hereditarily minimal cover refineable space is a hereditary Lindelöf space.

COROLLARY 2.10. A necessary and sufficient condition that a hereditarily separable minimal cover refineable space be hereditarily minimal cover refineable is that the space be perfectly normal.

THEOREM 2.11. For every collectionwise Hausdorff Moore space, the weight is equal to the Soustin number.

Pro of. Let $S$ be a.Moore space, $\left\{G_{i}\right\}$ a sequence which is a development for $S$, and $B$ a collection of $m$ many open sets which is a basis for $S$ where $m$ is the weight of the space. Only the case where $m \geqslant s_{0}$ is considered. For each integer $i>0$, let $G_{i}^{\prime}$ be a refinement of $G_{i}$ which is a minimal cover of $S$ and let $P_{i}$ be a collection containing one and only one point from each element of $G_{i}^{\prime}$ which is in no other element of $G_{i}^{\prime}$ Since $\left\{G_{i}^{\prime}\right\}$ is also a development of $S$, the cardinality of $\left\{g: i>0, g \in G_{i}^{\prime}\right\}$ is not less than $\mathrm{m}$. If $\mathrm{n}<\mathrm{m}$, then for some integer $I>0$, the cardinality of $G_{I}^{\prime}$ is not less than $\pi$. Let $H_{I}$ be a collection of disjoint open sets covering $P_{I}$ such that no element of $H_{I}$ contains two points of $P_{I}$. Then the Souslin number of $S$ is not less than the cardinality of $H_{I}$ nor more than the weight of $S$.

An example of a space which contains a dense metric subspace but is not minimal cover refineable is provided by $[0, \Omega)$. Following are two examples of stratifiable spaces which are not semi-metric. Note that stratifiable spaces are paracompact.
Let $S$ consist of one copy of the plane for each countable ordinal. If $P_{a}$ and $P_{\beta}$ are two of these planes in $S$, let $P_{a} \cap P_{\beta}=(0,0)$. Let basis elements for a point $p$ of $S$ distinct from $(0,0)$ be any open set in the plane containing $p$ which does not intersect $(0,0)$. For each countable ordinal $\alpha$ and each integer $i>0$, let a basis element for the point $(0,0)$ consist of the point $(0,0)$ plus the points in open disks of radius $1 / i$ with center at $(0,0)$ in each plane associated with an ordinal greater than $\alpha$.

The previous example suggested the following one to D. R. Traylor. Let $S$ be the points of a simple sequence of copies of the plane, $\left\{P_{i}\right\}$. For each pair of integers $i, j>0, i \neq j$, let $P_{i} \cap P_{j}=(0,0)$. For each integer $i>0$, let basis elements for $P_{i}$ be any open set in the plane that does not intersect the point $(0,0)$. Let each basis element for the point $(0,0)$ be the union of an open disk of the plane $P_{i}$ with radius $1 / J_{i}$ and center at $(0,0)$ for each integer $i>0$ where $J_{i}$ is a positive integer.

McAuley [27] gives an example of a stratifiable semi-metric space which is not developable.

3. Minimal cover refineable subsets. This section will be concerned with subsets of minimal cover refineable spaces. The section begins with the following obvious theorem:

THEOREM 3.1. Every closed subset of a metacompact or $F_{\sigma}$-screenable space is minimal cover refineable.

The statement that a collection $B$ of closed subsets of a space $S$ dominates $S$ means that $B$ covers $S$ and if $A C S$ and $A$ has a closed intersection with every element of some subcollection of $B$ which covers $A$, then $A$ is closed.

The concept of a dominating collection is due to Michael [28].

THEOREM 3.2. In order for every closed subset of a space to be minimal cover refineable, it is necessary and sufficient that the space be dominated by a collection of subsets such that every proper closed subset of an element of the collection is minimal cover refineable.

Proof. For the reason noted by Michael [28], necessity is obvious. Let $G$ be a collection of subsets which dominates a space $S$ such that every proper closed subset of an element of the collection is minimal cover refineable. Let $C^{\prime}$ be any open cover of $S$. Let $g$ be an element of $G$ and $c$ an element of $d^{\prime}$ which covers a point of $g$. Then $g \backslash c$ is minimal cover refineable, so $g$ is minimal cover refineable. Let $M$ be any closed su bset of $S$. Well-order the collection $\left\{g: g^{\prime} \in G\right.$ and $\left.g=g^{\prime} \cap M\right\}$ and call it $G^{\prime}$. Let $C$ be any open cover of $M$. Let $C_{1}$ be a refinement of $C$ which is a minimal cover of $g_{1}$, the first element of $G^{\prime}$. Let $C_{2}$ be a refinement of $\left\{c: c^{\prime} \in C\right.$ and $\left.c=c^{\prime} \backslash g_{1}\right\}$ which is a minimal cover of $g_{2} \backslash C_{1}^{*}$ where $g_{2}$ is $t$ the first element of $G^{\prime}$ not covered by $C_{1}^{*}$. After each initial segment, 
let $g_{\alpha}$ be the first element of $G^{\prime}$ not covered by $\bigcup C_{\beta}^{*}$ already constructed. Let $C_{\alpha}$ be a refinement of $\left\{c: c^{\prime} \in C\right.$ and $\left.c=c^{\prime} \backslash \bigcup_{\beta<a}^{\beta<a} g_{\beta}\right\}$ which is a minimal cover of $g_{\alpha} \backslash \cup C_{\beta}^{*}$. Then $\left\{c: a>0\right.$ and $\left.c \in C_{\alpha}\right\}$ is a minimal cover of $M$.

COROLLARX 3.3. Every open subset. of a space which has the property that every closed subset is $G_{\delta}$ and minimal cover refineable is minimal cover refineable.

THEOREM 3.4. Every open subset of a perfectly normal minimal cover refineable space is minimal cover refineable.

Proof. The following argument seems unnecessarily complicated. However, no simplification is apparent. Let $R$ be a proper open subset of $S$, a perfectly normal minimal cover refineable space, and $C$ any open cover of $R$. Since $S$ is perfectly normal, let $\left\{G_{i}\right\}$ be a sequence of open sets such that $\ \backslash R=\bigcap_{i=1}^{1 \infty} G_{i}=\bigcap_{i=1}^{\infty} \overline{G_{i}}$. Let $C^{\prime}=\left\{g: g^{\prime} \in O\right.$ and $\left.g=g^{\prime} \cap R\right\}$.

Let $C_{1}=\left\{g: g \in C^{\prime}\right.$ or $\left.g=G_{1}\right\}$. Let $C_{1}^{\prime}$ be a refinement of $C_{1}$ which is a minimal cover of $S$. Let $C_{1}^{\prime \prime}=\left\{g: g \in O_{1}^{\prime}\right.$ and $\left.g \not \subset G_{1}\right\}$. Let $M_{1}$ be the collection of all points of $\left(C_{1}^{\prime \prime}\right)^{*}$ which are not in two elements of $C_{1}^{\prime}$.

Let $C_{2}=\left\{g: g^{\prime} \in C^{\prime}\right.$ and $g=\left(g^{\prime} \cap G_{1} \backslash \backslash M_{1}, g=G_{2}\right.$, or $\left.g=\left(O_{1}^{\prime \prime}\right)^{*}\right\}$. Let $C_{2}^{\prime}$ be a refinement of $C_{2}$ which is a minimal cover of $S$. Let $C_{2}^{\prime \prime}$ $=\left\{g: g \in C_{2}^{\prime}\right.$ and $\left.g \nsubseteq\left(G_{2} \cup\left(C_{1}^{\prime \prime}\right)^{*}\right)\right\}$. Let $M_{2}$ be the collection of all points of $\left(C_{2}^{\prime \prime}\right)^{*}$ which are not in two elements of $C_{2}^{\prime}$.

For each integer $i>2$, let

$$
C_{i}=\left\{g: g^{\prime} \in C^{\prime} \text { and } g=\left(g^{\prime} \cap \bigcap_{j=1}^{i-1} G_{j}\right) \backslash \bigcup_{j=1}^{i-1} M_{j}, g=G_{i} \text {, or } g=\bigcup_{j=1}^{i-1}\left(C_{j}^{\prime \prime}\right)^{*}\right\} \text {. }
$$

Let $C_{i}^{\prime}$ be a refinement of $C_{i}$ which is a minimal cover of $S$. Let

$$
C_{i}^{\prime \prime}=\left\{g: g \in C_{i}^{\prime} \text { and } g \not \subset\left(G_{i} \cup \bigcup_{j=1}^{i-1}\left(C_{j}^{\prime \prime}\right)^{*}\right\}\right.
$$

Let $M_{i}$ be the collection of all points of $\left(C_{i}^{\prime \prime}\right)^{*}$ which are not in two elements of $C_{i}^{\prime}$.

Let $C_{0}=\left\{g: i>0 \quad g^{\prime} \in C_{i}^{\prime \prime}\right.$ and $\left.g=g^{\prime} \bigcup_{j=i+1}^{\infty} M_{j}\right\}$. By its construction, $C_{0}$ is a minimal cover of $R$.

Since the properties named are hereditary in a space in which closed sets are $G_{\delta}$, the following theorem is true:

THEOREM 3.5. If a space has the property that closed sets are $G_{\delta}$ and is either metacompact or $F_{\sigma}$-screenable, then the space is hereditarity minimal caver refineable.

An example of a paracompact space which is not hereditarily minimal cover refineable is given by $[0, \Omega]$. The following is an example of a minimal cover refineable space which contains a closed set which is not minimal cover refineable. It is based on a suggestion by $\mathrm{C}$. W. Proctor.

Let $T$ be the points of $[0, \Omega)$. For each point $p$ of $[0, \Omega)$, let $T_{p}$ be an independent copy of $[0, \Omega)$ and let $T_{p}$ have the order topology. Let $S=\left\{p: p \in T\right.$ or $q \in T$ and $\left.p \in T_{q}\right\}$. For each point $p$ of $T$, let an open set containing $p$ be the union of all the points between $q$ and $r$ where $q, r \in T$ and $q<p<r$ (for 0 , use $[0, r)$ ) plus the union of all the points. greater than some point of $T_{s}$ for each $s \in T$ such that $q<s<r$.

\section{References}

[1] P. Alexandroff, On some results concerning topological spaces and their continuous mappings, pp. 41-54 in General Topology and Its Relations to Modern Analysis and Algebra, Proceedings of the Prague Topological Symposium, 1961, New York 1963.

[2] B. A. Anderson, Topologies comparable to metric topologies, Topology Conference, Arizona State University, Arizona 1967, pp. 15-21.

[3] G. Aquaro, Point countable open coverings in countably compact spaces, pp. 39-41 in General Topology and Its Relations to Modern Analysis and Algebra II, Proceedings of the Second Prague Topological Symposium, 1966, New York 1967.

[4] R. Arens, and J. D ugundji, Remarls on the concept of compactness, Portugaliae Math. 9 (1950), pp. 141-143.

[5] A.Arhangel'skiř, IMappings and spaces, Russian Math. Surveys 21 (1966), pp. 87-114.

[6] R. H. Bing, Metrization of topological spaces, Canad. J. Math. 3 (1951), pp. 175-186.

[7] C. J. R. Borges, On stratifiable spaces, Pacific J. Math. 17 (1966), pp. 1-16.

[8] E. Cech, Topological Spaces, New York 1966.

[9] J. G. Ceder, Some generatizations of metric spaces, Pacific J. Math. 11 (1961), pp. 105-125.

[10] H. H. Corson, and E. Michael, Metrizability of certain countable unions, Illinois J. Math. 8 (1964), pp. 351-360.

[11] G. D. Creede, Ooncerning semi-stratifiable spaces, Pacific J. Math. 32 (1970), pp. 47-54.

[12] - Semi-stratifiable spaces, Topology Conference, Arizona State University, Tempe, Arizona 1967, pp. 318-323.

[13] J. Dugundji, Topology, Boston 1966.

[14] B. Fitzpatrick, On dense subspaces of Moore spaces, Proc. Amer. Math. Soc. 16 (1965), pp. 1324-1328.

[15] - On dense subspaces of Moore spaces II, Fund. Math. 61 (1967), pp. 91-92.

[16] E. E. Grace, and R. W. Heath, Separability and metrizability in pointwise paracompact Moore spaces, Duke Math. J. 31 (1964), pp. 603-610.

[17] R. W. Heath, On certain first countable spaces, Topology Seminar, Wisconsin 1965 , Annals of Math. Studies, 60, Princeton 1966, pp. 103-113.

18] - On open mappings and certain spaces satisfying the first conntability axiom, Fund. Math. 57 (1965), pp. 91-96.

[19] - A paracompact semi-metric space which is not an $M_{3}$-space, Proc. Amer. Math. Soc. 17 (1966), pp. 868-870.

[20] - Screenability, pointwise paracompactness, and metrization of Moore spaces, Canad. J. Math. 16 (1964), pp. 763-770. 

[21] - Semi-metric spaces and related spaces, Topology Conference, Arizona State
University, Tempe, Arizona 1967, pp. 153-161.

[22] J. L. Kelley, General Topology, Princeton 1959.

[23] R. G. Lubben, Separabilities of arbitrary orders and related properties, Bull. Amer. Math. Soc. 46 (1940), pp. 913-919.

[24] L. F. McAuley, A note on complete collectionwise normality and paracompactness, Proc. Amer. Math. Soc. 9 (1958), pp. 796-799.

[25] - A note on naturally ordered sets in semi-metric spaces, Proc. Amer. Math. Soc. 8 (1957), pp. 384-386.

[26] - On semi-metric spaces, Summer Institute on Set-Theoretic Topology, Madison, Amer. Math. Soc., 1955, pp. 60-64.

[27] - A relation between perfect separability, completeness, and normality in semimetric spaces, Pacific J. Math. 6 (1956), pp. 315-326.

[28] E. Michael, Continuous selections I, Ann. of Math. 63 (1956), pp. 361-382.

[29] - A note on paracompact spaces, Proc. Amer. Math. Soc. 4 (1953), pp. 831-838. [30] K. Morita, Star-finite coverings and the star-finite property, Math. Japonicae
1 (1948), pp. 60-68.

[31] J. Nagata, A contribution to the theory of metrization, J. Inst. Polytech., Osaka City University 8 (1957), pp. 185-192. [32] C. W. Proctor, Metrizable subsets of Moore spaces, Fund. Math. 66 (1969),
pp. 85-93.

[33] A. H. Stone, Cardinals of closed sets, Mathematika 6 (1959), pp. 99-107. [34] - Paracompactness and product spaces, Bull. Amer. Math. Soc. 54. (1948)
pp. 977-982.

[35] J. M. Worrell, Jr., and H. H. Wicke, Characterizations of developable topological spaces, Canad. J. Math. 17 (1965), pp. 820-830.

[36] J. N. Younglove, Concerning dense metric subspaces of certain non-metric spaces,
Fund. Math. 48 (1959), pp. 15-25.

Regu par la Rédaction le 2\%. 4. 1971

\section{On Helling cardinals $\left(^{(1)}\right.$}

by

\section{A. Wojciechowska (Wrocław)}

$\S$ 0. Introduction. The cardinal number $\varkappa>\omega_{1}$ is called a Helling cardinal if every relational structure with a $x$-like ordering has an elementary substructure with a $\lambda$-like ordering for an arbitrary $\omega_{0}<\lambda<\varkappa$. (If the language $\boldsymbol{L}$ of the structure under consideration is uncountable, then we can modify this notion, requiring $\lambda>|L|$.)

The existence of Helling cardinals is incompatible with the Axiom of Constructibility: it is easy to see that if there exists a Helling cardinal then $P\left(\omega_{0}\right) \cap L$ is countable. By the method of Silver [9] one can obtain the existence of $O^{\#}$ provided that there exists a Helling cardinal.

In 1966 Helling [2] proved that every measurable cardinal is a Helling cardinal, and Silver [8] has shown the same for Ramsey cardinals. These results seem to imply that Helling cardinals are very large. In Section 2 there is given a sufficient condition for a cardinal to be a Helling cardinal. This condition is satisfied by - among others - real-valued measurable cardinals. Prikry's result [6] entails that cardinals fulfilling our condition may be less than the continuum.

From the example of Fuhrken [1] we know that every Helling cardinal $*$ must be regular. If there is a limit (strongly limit) cardinal $\omega_{0}<\lambda<x$, then $x$ is weakly (strongly) inaccessible - see Keisler [3]. From these remarks it is clear that every Helling cardinal which is larger than $\omega_{\omega}$ must be weakly inaccessible. The question arises: can $\omega_{n}$ be a Helling cardinal? A partial solution of this is given in Section 3 .

In Section 4 we give an application of previous results to a language with a generalized quantifier $\left({ }^{2}\right)$.

(1) The results of this paper are contained in the doctoral dissertation prepared by the authoress under the guidance of Professor C. Ryll-Nardzewski at Wroclaw University. See also [11].

(2) The Corollary from [11] is not stated here. $I$ am indebted to Professor A. Mosto wski, who pointed out a gap in that proof. If this theorem is still true, the proof will be published. 